\title{
Phytoprotection
}

\section{Lien entre la préférence d'oviposition et la performance subséquente des larves chez la mineuse des agrumes Phyllocnistis citrella Stainton [Lepidoptera : Gracillariidae]}

\section{Saida Kharrat et Abderrahmane Jarraya}

Volume 86, numéro 1, avril 2005

URI : https://id.erudit.org/iderudit/011711ar

DOI : https://doi.org/10.7202/011711ar

Aller au sommaire du numéro

Éditeur(s)

Société de protection des plantes du Québec (SPPQ)

ISSN

0031-9511 (imprimé)

1710-1603 (numérique)

Découvrir la revue

Citer cet article

Kharrat, S. \& Jarraya, A. (2005). Lien entre la préférence d'oviposition et la performance subséquente des larves chez la mineuse des agrumes

Phyllocnistis citrella Stainton [Lepidoptera : Gracillariidae]. Phytoprotection, 86(1), 25-29. https://doi.org/10.7202/011711ar
Résumé de l'article

La femelle de la mineuse des agrumes Phyllocnistis citrella [Lepidoptera : Gracillariidae] recherche de façon préférentielle les jeunes feuilles, riches en éléments nutritifs, pour pondre ses oeufs et délaisse les feuilles âgées. La femelle a aussi tendance à revenir sur un hôte où un dépôt d'oeufs en surnombre a préalablement été effectué par d'autres femelles. Il s'en suit une forte mortalité larvaire. Toutefois, la taille des survivants n'est pas affectée parce que la régulation se fait par élimination des individus surnuméraires.
Tous droits réservés @ C La société de protection des plantes du Québec, 2005
Ce document est protégé par la loi sur le droit d'auteur. L'utilisation des services d'Érudit (y compris la reproduction) est assujettie à sa politique d'utilisation que vous pouvez consulter en ligne.

https://apropos.erudit.org/fr/usagers/politique-dutilisation/ 


\title{
Lien entre la préférence d'oviposition et la performance subséquente des larves chez la mineuse des agrumes Phyllocnistis citrella Stainton [Lepidoptera : Gracillariidae]
}

\author{
Saida Kharrat et Abderrahmane Jarraya'
}

Reçu le 2005-05-20; accepté le 2005-08-25

PHYTOPROTECTION 86 : 25-29

La femelle de la mineuse des agrumes Phyllocnistis citrella [Lepidoptera : Gracillariidae] recherche de façon préférentielle les jeunes feuilles, riches en éléments nutritifs, pour pondre ses œufs et délaisse les feuilles âgées. La femelle a aussi tendance à revenir sur un hôte où un dépôt d'œufs en surnombre a préalablement été effectué par d'autres femelles. II s'en suit une forte mortalité larvaire. Toutefois, la taille des survivants n'est pas affectée parce que la régulation se fait par élimination des individus surnuméraires.

Mots clés : Oviposition, Phyllocnistis citrella, surface foliaire, taille des chrysalides.

[Relationship between oviposition preference and the subsequent performance of citrus leafminer Phyllocnistis citrella [Lepidoptera: Gracillariidae] larvae]

The female citrus leafminer Phyllocnistis citrella [Lepidoptera: Gracillariidae] principally favours young leaves that are the richest in nutritive elements to lay eggs and leaves aside the oldest ones. Females also tend to come back to a host already infested with supernumerary egg deposition by other females. The result of this behaviour is a high larval mortality, but the size of the survivors is not affected as regulation occurs through the elimination of supernumerary individuals.

Keywords: Leaf surface, oviposition, Phyllocnistis citrella, size of pupae.

L'influence du site de ponte sur le rendement de la progéniture est un des facteurs pouvant modeler de façon évolutive le choix d'un site d'oviposition. Le choix du site de ponte chez certains insectes peut aussi influencer la valeur adaptative (fitness) des femelles. Chez la mineuse des agrumes, Phyllocnistis citrella Stainton, les œufs sont déposés sur les feuilles des agrumes et le développement larvaire s'effectue à l'intérieur d'une galerie sous corticale, creusée par ces dernières à l'intérieur d'un seul organe. Le choix du site de ponte des femelles $P$. citrella représente donc un élément déterminant pour la survie de la progéniture. Phyllocnistis citrella peut s'attaquer à plusieurs espèces et variétés d'agrumes avec une préférence pour le citronnier et à moindre degré les orangers et le mandarinier (Batra et Sandhu 1981; Batra et al. 1992; Singh et al. 1988; Thompson 1988; Verma 1989). La préférence peut également varier selon les individus d'un même cultivar et pour les organes d'une même plante-hôte. L'insecte est capable de sélectionner les sites les plus favorables pour le développement de sa progéniture.
Le processus de sélection chez les insectes est guidé en général par plusieurs stimuli visuels (Prokopy et Owens 1983) ou olfactifs chez la mineuse des agrumes (Wilson 1991). Selon Kennedy (1965), la sélection de la plante-hôte par un insecte est déterminée par des substances chimiques produites par la plante, lesquelles peuvent avoir un effet attractif ou répulsif, ce qui aide l'insecte à orienter son choix.

Pour ce qui est de la mineuse des agrumes (espèce multivoltine), la femelle a tendance à choisir les feuilles jeunes se trouvant dans la partie apicale du rameau (Jarraya et al. 1997). Les feuilles plus âgées à parenchyme plus ou moins lignifié, sont pratiquement délaissées. Par ce comportement, les femelles cherchent à optimiser les chances de survie de leur progéniture puisque les feuilles jeunes sont de meilleure qualité pour l'alimentation des insectes (Dixon 1985). Ce site de ponte peut par contre faire place à de la surpopulation et ainsi présenter des risques en termes de compétition pour la nourriture et de mortalité larvaire. 1. Département d'entomologie, Institut National Agronomique, Cité Mahrajène, 1082 Tunis, Tunisie; auteur auquel la correspon-
dance doit être adressée : kharratsaida@yahoo.fr 
Dans un programme de lutte biologique, il est nécessaire pour l'élevage d'insectes de s'assurer des meilleures conditions de croissance larvaire (rapport feuilles réceptives/femelles pondeuses) en perspective d'une optimisation de la production d'agents biologiques performants.

La présente étude se propose d'analyser dans quelle mesure le comportement de ponte de la mineuse des agrumes peut être favorable ou défavorable à la survie et à la bonne croissance de sa progéniture. L'objectif principal est de mettre en relation (1) la surface foliaire et la densité de chrysalides, (2) la surface foliaire et la taille des chrysalides, et enfin (3) la taille et la densité de chrysalides.

L'étude a été réalisée dans un verger d'une superficie de 1 ha situé à Mraissa au Cap Bon Tunisien. La parcelle d'études comporte des citronniers Citrus limon (L.) Burm. f. cv. Eureka âgés de 10 ans et plantés avec des espacements de $3 \mathrm{~m}$ entre les arbres et de $4 \mathrm{~m}$ entre les rangées.

Pour étudier l'influence de l'âge des feuilles sur le comportement de ponte de P. citrella, 20 jeunes pousses automnales ont été prélevées sur 20 citronniers pris au hasard. Ces pousses renferment des feuilles tendres et partiellement développées. Le prélèvement a été effectué le 2 novembre 2002, période correspondant à une forte production de nouvelles pousses chez le citronnier. Les rameaux prélevés ont été examinés sous loupe binoculaire au laboratoire, en commençant par la partie terminale, afin de dénombrer les œufs et les jeunes larves s'y trouvant.

Les feuilles prélevées ont été groupées en classes selon leur longueur. Ces classes rassemblent des feuilles ayant des longueurs rapprochées, notre hypothèse de travail étant que la longueur des feuilles est un indicateur approximatif de l'âge de la feuille.

Pour examiner l'effet de la plante hôte sur la survie et la croissance de la descendance, un $2^{\mathrm{e}}$ prélèvement a été effectué dans le même verger 3 semaines plus tard (23 novembre). Cette période est requise afin de permettre I'achèvement du développement larvaire dans les conditions climatiques locales (Boulahia Kheder et al. 2002). Pour chacune des feuilles, la surface foliaire a été mesurée, le nombre de chrysalides présentes a été déterminé, de même que la taille de ces dernières.

La surface foliaire a été calculée selon la formule d'Onillon et al. (1971) mise au point pour les feuilles de bigaradier que nous avons appliquée aux feuilles de citronnier :

$S b=0.1025+0.6637 a . b$

$S=$ surface foliaire réelle en $\mathrm{cm}^{2}$

$a=$ la plus grande longueur de la feuille en $\mathrm{cm}$

$b=$ la plus grande largeur de la feuille en $\mathrm{cm}$.

L'extension de cette formule aux feuilles de citronnier nous a paru possible, étant donné leur morphologie assez semblable à celle des feuilles du bigaradier. Les feuilles ont été groupées en quatre classes, selon l'importance de leur surface exprimée en $\mathrm{cm}^{2}$. Sur chacune des feuilles observées à l'intérieur d'une classe, le nombre de chrysalides était évalué.

Dans le but d'étudier l'impact du substrat de ponte sur le comportement d'oviposition de la mineuse des agrumes, nous avons procédé à une analyse de la variance à un seul facteur (classe) selon le procédé GLM du système SAS (2000). De plus, un test de Duncan pour les comparaisons multiples a été effectué afin de comparer les moyennes des différents traitements.

Une régression linéaire simple a aussi été utilisée pour corréler la surface foliaire avec la densité des chrysalides, la surface foliaire avec la taille des chrysalides et enfin la taille et la densité des chrysalides.

Les résultats obtenus ont permis de constater que la majorité des œufs sont déposés sur les feuilles très jeunes mesurant entre 1 et $3 \mathrm{~cm}$ de longueur ( $\mathrm{Ta}$ bleau 1). En effet, celles-ci portent $65,46 \%$ de la totalité des oeufs dénombrés. II est à noter que les femelles sont capables de déposer des œufs sur des feuilles dont la taille est inférieure à $1 \mathrm{~cm}$, mais avec un faible taux de ponte de $4,34 \%$.

Tableau 1. Répartition des œufs et jeunes larves de Phyllocnistis citrella selon la longueur des feuilles de citronnier

\begin{tabular}{ccccc}
\hline $\begin{array}{c}\text { Longueur } \\
\text { des feuilles } \\
\text { (cm) }\end{array}$ & $\begin{array}{c}\text { Nombre } \\
\text { de feuilles } \\
\text { observées }\end{array}$ & $\begin{array}{c}\text { Nombre } \\
\text { d'œufs + jeunes } \\
\text { larves }\end{array}$ & $\begin{array}{c}\text { Ponte } \\
(\%)\end{array}$ & $\begin{array}{c}\text { Densité } \\
\text { de la ponte/feuille } \\
\text { (moyenne } \pm \text { SEM) }\end{array}$ \\
\hline $0-1$ & 15 & 55 & 4,34 & $4,33 \pm 1,86 \mathrm{c}^{\mathrm{a}}$ \\
$1,1-2$ & 50 & 418 & 37,54 & $11,24 \pm 3,68 \mathrm{a}$ \\
$2,1-3$ & 41 & 158 & 27,92 & $10,19 \pm 3,69 \mathrm{a}$ \\
$3,1-4$ & 23 & 195 & 10,55 & $7,86 \pm 1,81 \mathrm{~b}$ \\
$4,1-5$ & 27 & 50 & 3,03 & $3,22 \pm 1,66 \mathrm{~b}$ \\
$5,1-6$ & 13 & 20 & 1,34 & $1,66 \pm 0,66 \mathrm{c}$ \\
$6,1-7$ & 12 & 22 & 1,47 & $2,75 \pm 1,28 \mathrm{~d}$ \\
$7,1-8$ & 8 & 4 & 0,27 & $0,66 \pm 0,51 \mathrm{e}$ \\
$8,1-9$ & 6 & 3 & 0,20 & $0,23 \pm 0,48 \mathrm{e}$ \\
$\geq 9,1$ & 13 & 1497 & 100 &
\end{tabular}

${ }^{a}$ Les moyennes suivies des mêmes lettres ne sont pas significativement différentes; seuil de $P<0,015$. 


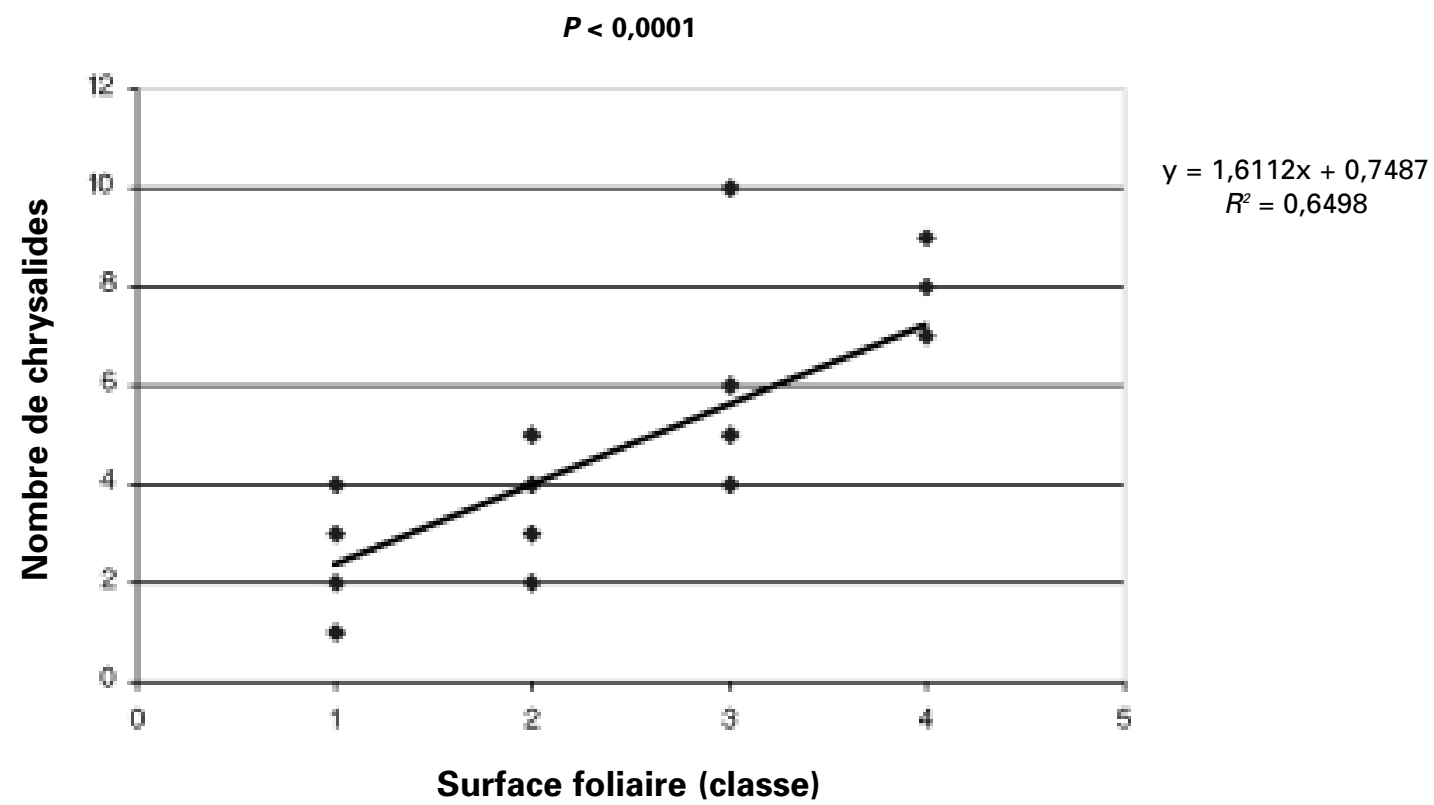

Figure 1. Relation entre la surface des feuilles de citronnier et le nombre de chrysalides de Phyllocnistis citrella.

La densité de ponte varie selon un gradient décroissant au fur et à mesure qu'on s'éloigne de l'apex du rameau $(P<0,015)$. Ceci signifie que la femelle $P$. citrella est capable de distinguer l'intensité de lignification de la feuille. Plus celle-ci est coriace, moins elle est recherchée par la femelle en quête d'un site de ponte. Par contre, aucune donnée ne nous permet de déterminer à ce jour si la perception par la femelle est de nature physique (topographique, dureté, etc.) ou chimique. Le choix préférentiel de la femelle pour les jeunes feuilles semble être dicté par des considérations nutritionnelles pour les larves dans la mesure où ces organes sont les plus riches en substances azotées, composés essentiels pour la croissance des larves.

L'analyse statistique montre qu'il existe une corrélation positive $\left(R^{2}=0,64\right)$ entre la surface foliaire et le nombre d'individus par feuille, c'est-à-dire que plus la surface de la feuille est importante, plus elle renferme un grand nombre de chrysalides $(y=1,6112 x+$ 0,7487; $P<0,0001$ ) (Fig. 1). Lors de l'étude sur I'effet du substrat végétal sur la survie de la mineuse, les feuilles examinées étaient les plus recherchées par les femelles en quête de ponte.
La comparaison des tableaux 1 et 2 montre que si la moyenne d'œufs déposés par feuille est de 7,2 œufs (1497/208), celle des chrysalides n'est en revanche que de 4,1 chrysalides (205/50). Ceci démontre que la progéniture de $P$. citrella est soumise à une mortalité de l'ordre de $45 \%$, vraisemblablement due à un déficit nutritionnel dans la mesure où les autres facteurs de mortalité (cannibalisme, stress climatique, action des antagonistes) sont absents. La présence de jeunes larves à l'intérieur de galeries foliaires n'arrête pas la croissance de la feuille, exceptée lors d'une surpopulation, ce qui conduit à la fois à la mort du support végétal et des jeunes larves.

Il a été possible de démontrer que la survie des chrysalides est plus élevée lorsque que la surface foliaire est grande. Bell (1991) rapporte qu'il existe une grande corrélation entre le choix de l'hôte par la femelle et la performance larvaire. Par contre, l'âge de la feuille constitue un autre paramètre pouvant affecter la croissance des larves de la mineuse des agrumes. Il faut que les feuilles réceptives soient relativement de grande dimension. Dans le cas contraire (feuilles de petite taille), le phénomène de surpopulation se traduit par une destruction plus ou moins

Tableau 2. Densité des chrysalides sur les 50 feuilles de citronnier examinées

\begin{tabular}{cccc}
\hline $\begin{array}{c}\text { Surface foliaire } \\
\left(\mathrm{cm}^{2}\right)\end{array}$ & $\begin{array}{c}\text { Nombre de } \\
\text { feuilles observées }\end{array}$ & $\begin{array}{c}\text { Nombre } \\
\text { de chrysalides }\end{array}$ & $\begin{array}{c}\text { Densité } \\
\text { chrysalides/feuille } \\
(\mathrm{n} \pm \text { SEM) }\end{array}$ \\
\hline $4,5-10,4$ & 16 & 37 & $2,3 \pm 1,01$ \\
$10,5-16,6$ & 17 & 58 & $3,4 \pm 0,71$ \\
$16,8-22,8$ & 13 & 32 & $6,0 \pm 1,56$ \\
$\geq 24$ & 4 & 205 & $8,0 \pm 0,81$ \\
Total & 50 & 32 & \\
\hline
\end{tabular}


$P=0,74$

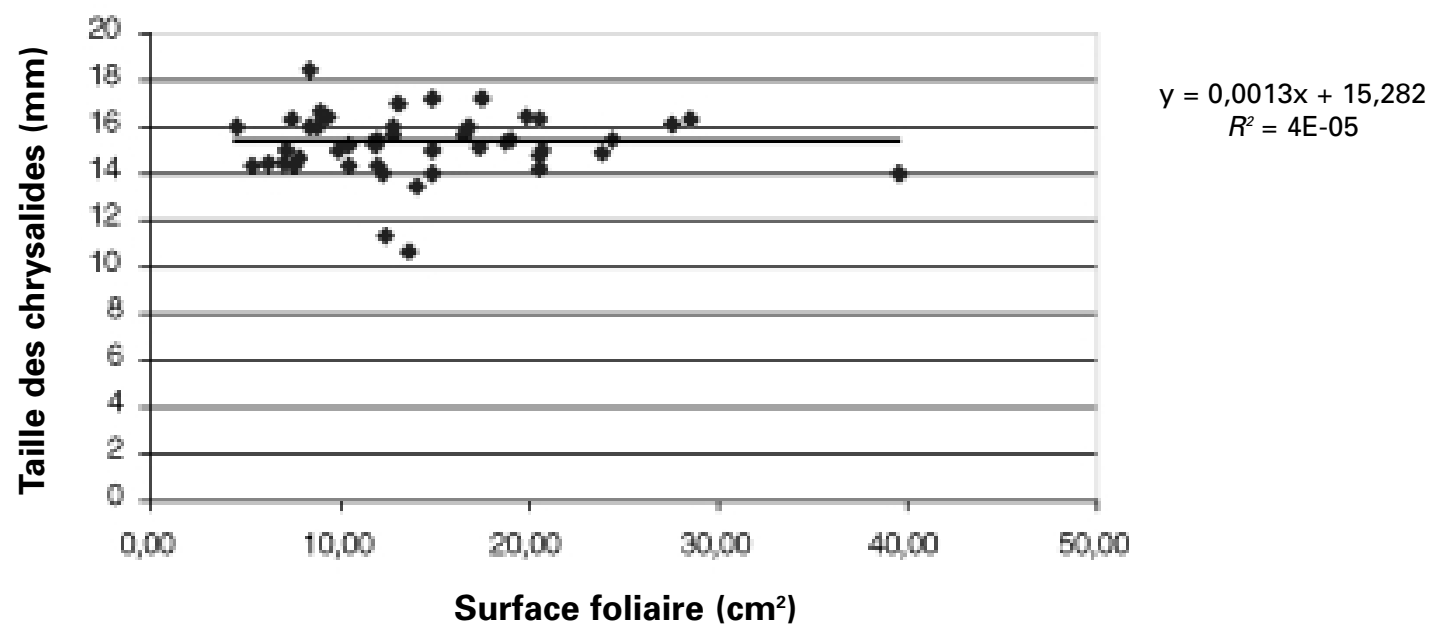

Figure 2. Relation entre la surface des feuilles de citronnier et la taille moyenne des chrysalides de Phyllocnistis citrella.

complète du limbe avec pour conséquence la mort des individus qui lui sont associés.

Comme la surface foliaire semble avoir un effet direct sur la survie de la descendance de la mineuse, il serait intéressant de voir si elle influence la taille des chrysalides s'y trouvant. Puisque la performance d'une colonie de parasitoïdes élevés sur son insecte hôte dépend en grande partie de la quantité de nourriture offerte (Jarraya 1975), nous assumons que la dimension des feuilles pourrait influencer la taille des chrysalides de $P$. citrella.

L'analyse statistique montre que la taille des chrysalides n'est pas influencée par les dimensions du support végétal sur lequel elles se trouvent $(\mathrm{y}=$ $0,0013 x+15,282 ; P=0,74$ ) (Fig. 2). Ceci signifie que la feuille de Citrus, si elle n'est pas gravement affectée dans son intégrité, est en mesure d'offrir en quantité et en qualité suffisamment de nutriments pour assurer un développement normal de la mineuse. L'absence de corrélation négative entre l'âge de la feuille et la taille des chrysalides explique difficilement le comportement des femelles de $P$. citrella à rechercher de façon préférentielle les jeunes feuilles. II a aussi été possible d'observer l'absence de corrélation entre la taille des chrysalides et leur densité, c'est-à-dire que la taille des chrysalides n'est pas affectée par leur densité sur le support végétal ( $y=$ $0,00449 x+15,116 ; P=0,89$ ) (Fig. 3)]. Ceci suggère qu'il n'existe que très peu de compétition entre les larves lors de la recherche de nourriture. Lors de prochains travaux de recherche, il serait intéressant de vérifier si la taille des chrysalides peut être influencée par la composition biochimique des feuilles auxquelles elles sont associées.

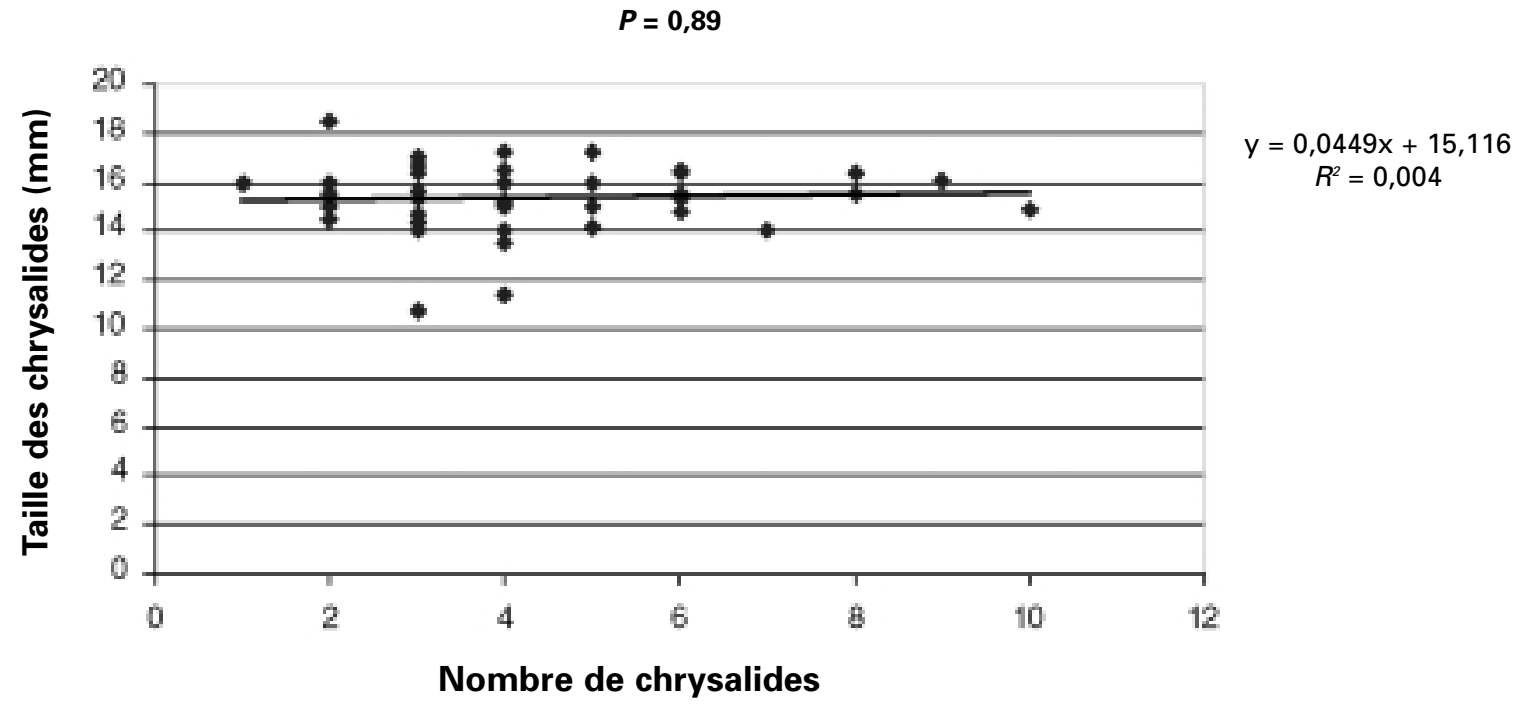

Figure 3. Relation entre la densité et la taille moyenne des chrysalides de Phyllocnistis citrella. 


\section{REMERCIEMENTS}

Nous adressons nos vifs remerciements à Monsieur Robert F. Luck, professeur d'entomologie à I'Université de Riverside en Californie; ses conseils judicieux et ses suggestions utiles ont été fort importants pour la réalisation de ce travail.

\section{RÉFÉRENCES}

Batra, R.C. et G.S Sandhu. 1981. Differential population of citrus leafminer and its parasite on some commercial Citrus cultivars. J. Res. Punjab Agric. Univ. 18 : 170-176.

Batra, R.C., D.R. Sharma et Y.R. Chanana. 1992. Screening of Citrus germplasm for their resistance against citrus leafminer, Phyllocnistis citrella Stainton. J. Insect Sci. 5 : 150-152.

Bell, W.J. 1991. Searching behaviour: the behavioural ecology of finding resources. Chapman and Hall, London. $358 \mathrm{pp}$.

Boulahia Kheder, S., A. Jarraya, F. Jrad et M. Fezzani. 2002. Étude de la mineuse des agrumes Phyllocnitis citrella Stainton (Lep. Gracillariidae) dans la région du Cap Bon (Tunisie). Fruits $57: 29-42$.

Dixon, A.F.G. 1985. Structure of aphid populations. Annu. Rev. Entomol. 30 : 155-174.

Jarraya, A. 1975. Contribution à l'étude des interactions hôte parasite chez Coccus hesperidium L. (Hom. Coccidae) et son parasite Coccophagus scutellaris DALM. (Hym. Aphelinidae). Thèse de Ph. D., Université de Paris VI. $86 \mathrm{pp}$.
Jarraya, A., S. Boulahia Kheder, F. Jrad et M. Fezzani. 1997. La mineuse des agrumes en Tunisie : bio-écologie et méthodes de lutte. Document technique, $20 \mathrm{pp}$.

Kennedy, J.S. 1965. Mechanisms of host plant selection. Ann. Appl. Biol. 56 : 317-322.

Onillon, J.C, J. Onillon et R. Tomassone. 1971. Contribution à l'étude de la dynamique des populations d'homoptères inféodés aux agrumes. Estimation de la surface d'une feuille en fonction de deux plus grandes dimensions. Ann. Zool. Ecol. Anim. 3 : 183-193.

Prokopy, R.J. et E.D. Owens. 1983. Visual detection of plants by herbivorous insects. Annu. Rev. Entomol. 28 : 337-364.

Singh, S.P., N.S. Rao, K.K. Kuma et B.S. Bhumannavar. 1988. Field screening of citrus germplasm against the citrus leafminer, Phyllocnistis citrella Stainton. Indian J. Entomol. $50: 69-75$.

Thompson, J.N. 1988. Evolutionary ecology of the relationship between oviposition preference and performance of offspring in phytophagous insects. Entomol. Exp. Appl. $47: 3-14$.

Verma, R.R. 1989. Studies on the tolerance of species and varieties of Citrus to the citrus leafminer, Phyllocnistis citrella Stainton. Agric. Sci. Dig. (Karnal) 9 : 31-33.

Wilson, C.G. 1991. Notes on Phyllocnistis citrella Stainton (Lepidoptera: Phyllocnistidae) attacking four citrus varieties in Darwin. J. Austr. Entomol. Soc. 30 : 77-78. 